\title{
Time Evolution of Floquet States in Graphene
}

\author{
F. Manghi $\mathbb{D}^{1,2}$ M. Puviani, ${ }^{1}$ and F. Lenzini' ${ }^{1}$ \\ ${ }^{1}$ Dipartimento di Scienze Fisiche, Informatiche e Matematiche, Università di Modena e Reggio Emilia, \\ Via Campi 213/A, I-41125 Modena, Italy \\ ${ }^{2}$ CNR-Institute of NanoSciences-S3, Italy
}

Correspondence should be addressed to F. Manghi; franca.manghi@unimore.it

Received 29 January 2018; Revised 18 April 2018; Accepted 20 June 2018; Published 2 August 2018

Academic Editor: Sergio E. Ulloa

Copyright (c) 2018 F. Manghi et al. This is an open access article distributed under the Creative Commons Attribution License, which permits unrestricted use, distribution, and reproduction in any medium, provided the original work is properly cited.

Based on a solution of the Floquet Hamiltonian we have studied the time evolution of electronic states in graphene nanoribbons driven out of equilibrium by time-dependent electromagnetic fields in different regimes of intensity, polarization, and frequency. We show that the time-dependent band structure contains many unconventional features that are not captured by considering the Floquet eigenvalues alone. By analyzing the evolution in time of the state population we have identified regimes for the emergence of time-dependent edge states responsible for charge oscillations across the ribbon.

If a time-periodic field is applied to electrons in a periodic lattice the Bloch theorem can be applied twice, both in space and in time. This is the essence of Floquet-Bloch theory [1-3] that has recently attracted a renewed interest for its ability to describe topological phases in driven quantum systems [47]. The discovery that circularly polarized light may induce nontrivial topological behaviour in materials that would be standard in static conditions [8-11] has opened the way to the realization of the so-called Floquet topological insulators, where a topological phase may be engineered and manipulated by tunable controls such as polarization, periodicity, and amplitude of the external perturbation.

When the field is applied for a sufficiently long time (pulse duration much larger than the field oscillation period) electrons reach a nonequilibrium steady state characterized by a periodic time-dependence of the wave functions and, consequently, of the expectation values of any observable $[12,13]$. In this paper we focus on this time-dependence, looking for the time evolution of some relevant quantities such as energy and charge density. How these characteristics affect the time behaviour of these observables will be our focus. We will consider the prototypical case of graphene that under the influence of circularly polarized light exhibits in its Floquet band structure the distinctive characteristics of a 2D Chern insulator, namely, a gap in 2D and linear dispersive edge states in $1 \mathrm{D}[9,11,14,15]$. These Floquet edge states are topologically protected and responsible for a quantized Hall conductance in the absence of a magnetic field [16-18], a remarkable realization of the so-called "quantum Hall systems without Landau levels" originally proposed by Haldane [19].

Under a periodic drive, the nonequilibrium steady states, solutions of the time-dependent Schrödinger equation

$$
\left(\widehat{H}(\mathbf{r}, t)-i \frac{\partial}{\partial t}\right) \psi_{\mathbf{k}}(\mathbf{r}, t)=0
$$

evolve in time as

$$
\psi_{\alpha \mathbf{k}}(\mathbf{r}, t)=e^{-i \epsilon_{\alpha}(\mathbf{k}) t} \phi_{\alpha \mathbf{k}}(\mathbf{r}, t)
$$

where $\phi_{\alpha \mathbf{k}}(\mathbf{r}, t)$ is periodic in time and $\epsilon_{\alpha}(\mathbf{k})$, the Floquet quasi-energies, are the eigenvalues of an effective Hamiltonian $\widehat{H}^{F} \equiv \widehat{H}-i(\partial / \partial t)$, the so-called Floquet Hamiltonian:

$$
\widehat{H}^{F} \phi_{\alpha \mathbf{k}}(\mathbf{r}, t)=\epsilon_{\alpha}(\mathbf{k}) \phi_{\alpha \mathbf{k}}(\mathbf{r}, t)
$$

Here $\widehat{H}(\mathbf{r}, t)$ is the full Hamiltonian of the driven system

$$
\widehat{H}(\mathbf{r}, t)=\widehat{H}_{0}(\mathbf{r})+\widehat{V}(\mathbf{r}, t)
$$


with $H_{0}(\mathbf{r})$ being the static Hamiltonian and $V(\mathbf{r}, t)$ being the external periodic driving. The factorization in (2) is exact and represents the temporal analogue of the Bloch theorem. In the following we will consider states described by (2) characterized by a single wave vector $\mathbf{k}$ and of a given Floquet quasi-energy $\epsilon_{\alpha}(\mathbf{k})$. With $\phi_{\alpha \mathbf{k}}(\mathbf{r}, t)$ being time-periodic it can be expressed as a Fourier series:

$$
\phi_{\alpha \mathbf{k}}(\mathbf{r}, t)=\sum_{n=-\infty}^{\infty} B_{\alpha n \mathbf{k}}(\mathbf{r}) e^{-i n \Omega t}
$$

where in turn $B_{\alpha n \mathbf{k}}(\mathbf{r})$ can be expanded on a complete set, for instance, on a localized basis:

$$
B_{\alpha n \mathbf{k}}(\mathbf{r})=\sum_{i}^{N} C_{i n}^{\alpha}(\mathbf{k}) \chi_{i}(\mathbf{r})
$$

with $i$ being a site index, $N$ being the number of sites in the unit cell, and $\chi_{i}(\mathbf{r})$ being the localized orbitals. In practice the Fourier expansion is truncated to include a finite number of modes, up to a sufficiently large $n_{\max }$ whose value depends obviously on $\Omega$. This allows formulating the eigenvalue problem in (3) in a standard matrix form whose eigenvalues turn out to be replicas of the static band structure with gaps opening at their crossing points $[1,11]$.

The field-free Hamiltonian of graphene is described in the tight-binding scheme with a single hopping parameter $J \simeq 2.8 \mathrm{eV}$ between nearest neighbor sites, reproducing the well-known Dirac-like valence and conduction bands [20]. In the presence of the oscillating field described by the vector potential $\mathbf{A}(t)$, the hopping between neighboring sites is modified according to Peierls' substitution [21, 22]:

$$
\widetilde{J}_{i j}(t)=J e^{i \mathbf{A}(t) \cdot\left(\mathbf{r}_{j}-\mathbf{r}_{i}\right)} .
$$

We are interested in the effects of reduced dimensionality, namely, on the gapless edge states that arise in graphene ribbons; we chose a zig-zag terminated ribbon 50 atoms wide (Figure 1). We consider two frequency values $\left(\Omega_{1}=5.5 \mathrm{eV}\right.$, $\left.\Omega_{2}=12 \mathrm{eV}\right)$ representative of the intermediate and large frequency regime $\left(\Omega_{1} / J \simeq 2, \Omega_{2} / J \simeq 4\right)$. We study also the effect of different amplitudes of the external vector potential ( $A_{0}=0.5$ and $A_{0}=1.0$ in units of the inverse carbon-carbon distance [7]). In Figure 2 we compare the Floquet quasienergies obtained for the honeycomb lattice in $2 \mathrm{D}$ and $1 \mathrm{D}$ exposed to a circularly polarized field $\mathbf{A}(t)=A_{0}(\cos (\Omega t) \hat{x}+$ $\sin (\Omega t) \widehat{y})$.

Panels (a), (c), (e), and (g) report the Floquet Projected Bulk Band Structure (FPBBS), namely, the Floquet eigenvalues obtained for the 2D lattice using the same unit cell of the ribbon and periodically repeated in both $\mathrm{x}$ and $\mathrm{y}$ (along the ribbon and perpendicular to it), in this way restoring the $2 \mathrm{D}$ translation symmetry. As currently done in standard surface physics [23], the projected bulk band structure allows identifying straightaway the energy regions that, prohibited in the bulk, can host localized states at the edges. Panels (b), (d), (f), and (h) of Figure 2 report the Floquet quasi-energies obtained in the $1 \mathrm{D}$ ribbon geometry clearly showing extra states in the $2 \mathrm{D}$ forbidden regions. These states go in pairs being localized either on the upper or on the lower edge of the ribbon. We notice that for the largest frequency $\Omega=12$ $\mathrm{eV}$ the effect of increasing $A_{0}$ is to widen the gap between bulk Floquet bands and to increase the edge state dispersion. Gapless edge states appearing around $k_{x}=1 / 2$ (in units of $2 \pi / a)$ exhibit for both values of $A_{0}$ the peculiar linear dispersion evocative of a nontrivial topological character [24-26]. The same dispersion exists also for $\Omega=5.5 \mathrm{eV}$ but now the structure of Floquet bands is more complex and extra edge states appear at different k-points and in other gaps and lenses of the FPBBS. Smaller field strengths would correspond to even smaller bulk gaps and less dispersive edge states.

It is interesting to compare these results with those obtained assuming a linear polarization, with the vector potential oscillating perpendicular to the ribbon length $(\mathbf{A}(t)=$ $\left.A_{0} \sin (\Omega t) \widehat{y}\right)$. As shown in Figure $32 \mathrm{D}$ Floquet bands are gapless independently of the field strength. Edge states appearing in the middle of the $1 \mathrm{D}$ Brillouin Zone (BZ) have no appreciable $\mathrm{k}$-dispersion in the same way as edge states in static conditions [27].

We may conclude this analysis of Floquet quasi-energies by noticing that only circularly polarized fields of sufficient strength may induce Floquet edge states with a significant linear dispersion. It is interesting now to go one step further and use the solution of the Floquet problem to obtain information on physical observables.

Even if Floquet quasi-energies are used to interpret the photon dressed electronic excitations probed by spectroscopic techniques such as pump-probe experiments and time-resolved photoemission $[7,28-30]$ their connection with other measurable quantities is somewhat indirect: Floquet quasienergies are time-independent eigenvalues of an auxiliary Hamiltonian and therefore cannot capture all the essential time-dependent physics. However Floquet eigenvalues and eigenvectors can be used to calculate expectation values exactly, thanks to the exact representation of the time-dependent wave functions (see (5)). In particular the time-dependent single particle energies of the driven system, defined as the expectation values of the time-dependent Hamiltonian over $\psi_{\mathbf{k}}(\mathbf{r}, t)$, can be expressed in terms of the Floquet eigenstates as follows:

$$
\begin{aligned}
E_{\alpha}(\mathbf{k}, t) \equiv\left\langle\psi_{\alpha \mathbf{k}}(\mathbf{r}, t)|\widehat{H}(\mathbf{r}, t)| \psi_{\alpha \mathbf{k}}(\mathbf{r}, t)\right\rangle \\
=\left\langle\psi_{\alpha \mathbf{k}}(\mathbf{r}, t)\left|i \frac{\partial}{\partial t}\right| \psi_{\alpha \mathbf{k}}(\mathbf{r}, t)\right\rangle \\
=\epsilon_{\alpha}(\mathbf{k}) \\
\quad+\sum_{n, m} e^{i(n-m) \Omega t} m \Omega \int B_{\alpha, n, \mathbf{k}}(\mathbf{r})^{*} \cdot B_{\alpha, m, \mathbf{k}}(\mathbf{r}) d \mathbf{r} .
\end{aligned}
$$

Figure 4 shows the time-dependent single particle energies of the $2 \mathrm{D}$ system calculated at k-vectors around the Dirac point for different strengths and frequencies of the circularly polarized external field. We choose to plot $E(\mathbf{k}, t)$ as a function of the unperturbed Bloch momentum $\mathbf{k}$ instead of the shifted momentum $\mathbf{k}-\mathbf{A}(t)$ that takes into account the effect of the external field as shown in [31]. This shift 


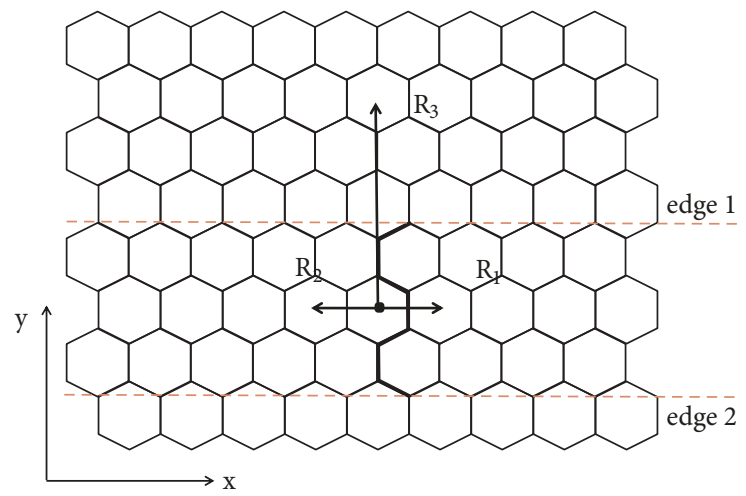

Figure 1: Geometry of a zig-zag honeycomb ribbon. Heavy lines indicate the unit cell for an 8-atom wide ribbon. $R_{1}, R_{2}$ are the lattice vectors associated with $1 \mathrm{D}$ translation symmetry. $R_{3}$ is the lattice vector to be used to reproduce the $2 \mathrm{D}$ lattice starting from the present unit cell (see text).

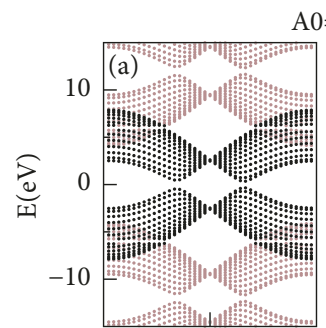

$\mathrm{A} 0=0.5$
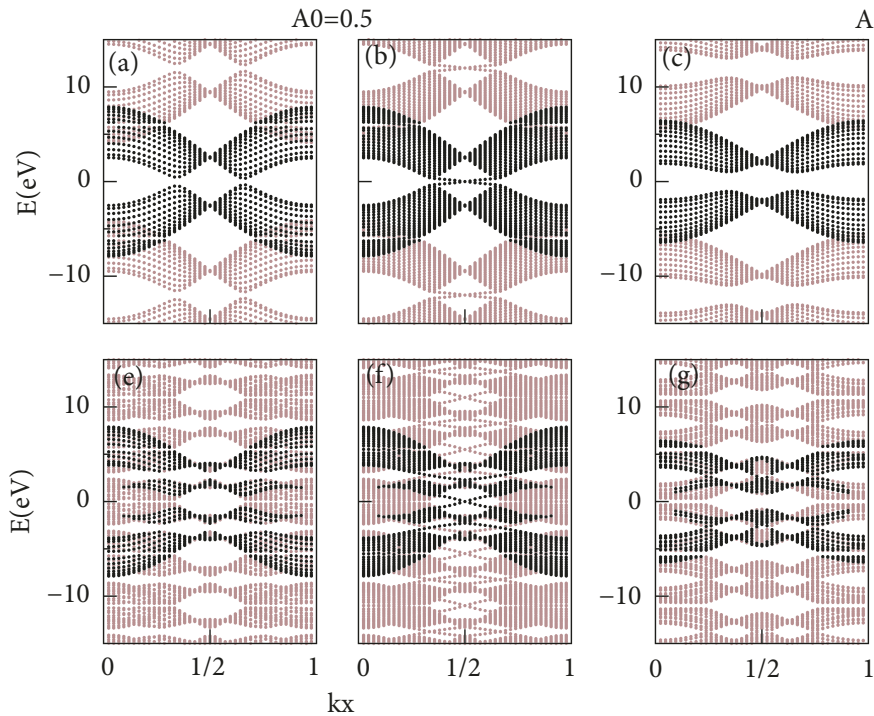

$\mathrm{A} 0=1$
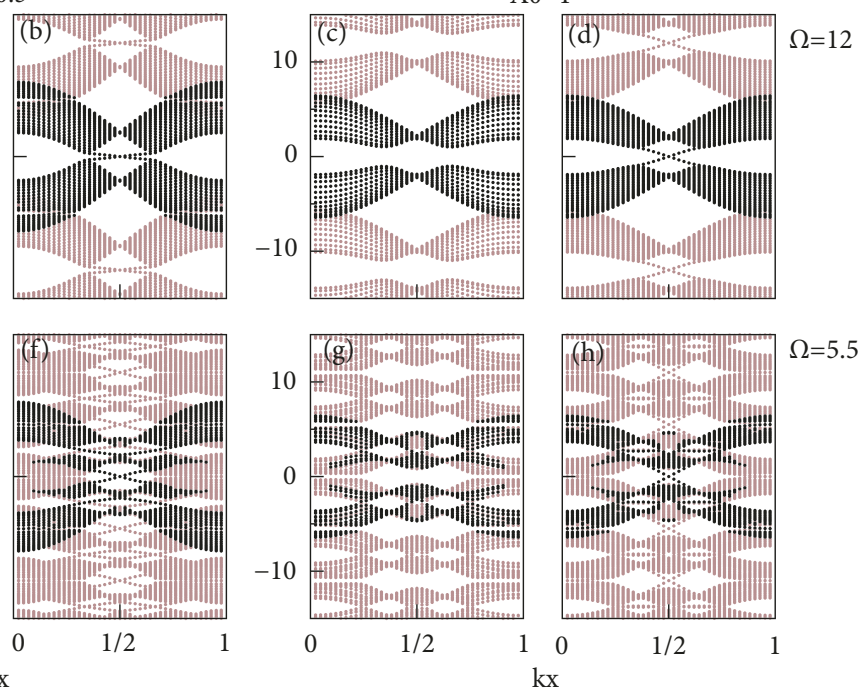

FIgURE 2: Floquet quasi-energies for circularly polarized field of different strengths and frequencies. Panels (a), (c), (e), and (g) report the Floquet Projected Bulk Band Structure, panels (b),(d), (f), and (h) report the results for the zig-zag ribbon. The full Floquet band structure (brown dots) corresponds to a replication of the zero-mode Floquet band (black dots). Upper panels: $\Omega=12 \mathrm{eV}$; lower panels $\Omega=5.5 \mathrm{eV}$; panels (a), (b), (e), and (f): $A_{0}=0.5$; panels (c), (d), (g), and (h): $A_{0}=1$.

is crucial when performing theoretical simulation of timeresolved photoemission experiments [32] but is not relevant when time-dependent energies are used for calculations that involve a summation over $k$ (see the following).

We show few snapshots at selected times $t_{n}=(n-1) / 8 T$ within the interval $0 \leq t \leq T / 2, T$ being the period of the external field. Since $E_{\alpha}(\mathbf{k}, t)=E_{\alpha}(\mathbf{k}, T-t)$ these snapshots are illustrative of the full time evolution. It is interesting to notice that the time-dependent energies $E_{\alpha}(\mathbf{k}, t)$, in spite of $\alpha$ running over both Floquet modes and band index, consist of two bands only since different Floquet modes give rise to the same time-dependent energy.

This is a consequence of the Floquet eigenvalues periodicity and while Floquet eigenvalues are defined modulo$\Omega$, time-dependent band energies are identified by the band index only.
A comment on the relationship between Floquet eigenvalues and time-dependent band energies $E(\mathbf{k}, t)$ is in order. The exponential prefactor in (2) is the envelope function of the full solution of the time-dependent Schrödinger equation. Thus Floquet quasi-energies that enter that term provide information on the amplitude modulation in time of the complete wave function, without catching the full time evolution but only the slowly varying one. Indeed, they correspond to the exact dynamics only in a stroboscopic picture, i.e., for multiple integers of the driving period. On the other hand, time-dependent energies show the complete time evolution of the solution of the Schrödinger equation, even the fastest components. Real experiments are in general sensitive only to the slowest dynamics; therefore real time-dependent energies become somehow elusive to a direct investigation. Nevertheless, as it will be pointed out in the following, other relevant 

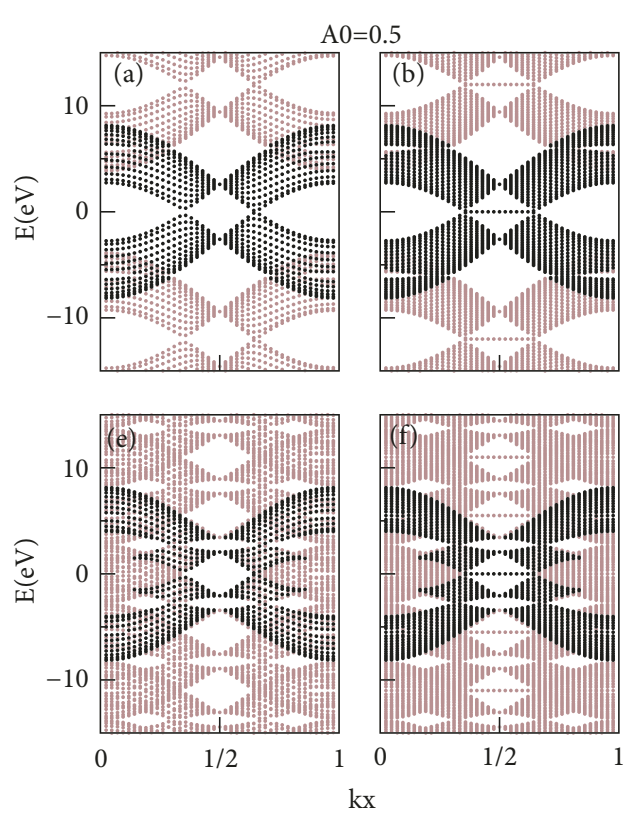

$\mathrm{A} 0=1$
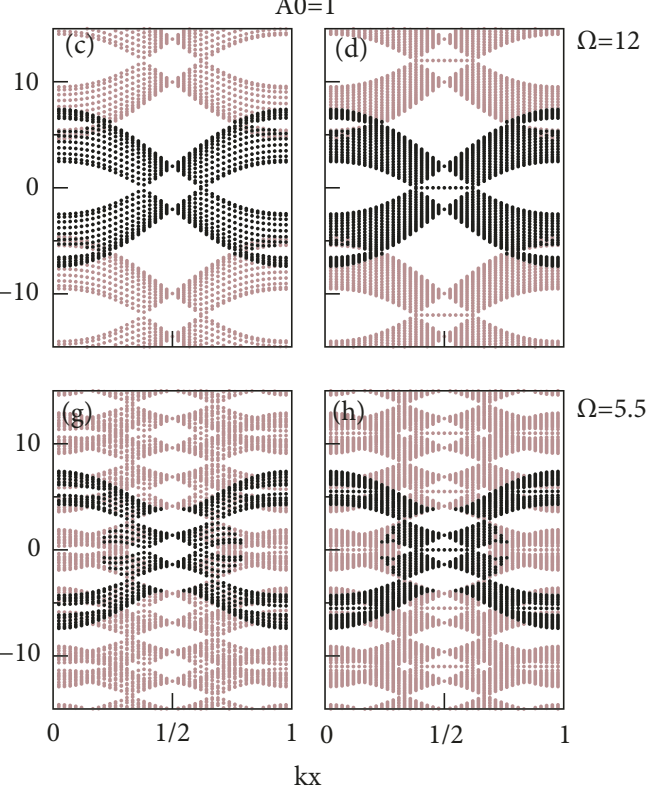

FIGURE 3: Same as Figure 2 but for linear polarization.

and observable quantities such as charge and currents can be truly evaluated only considering the full time-dependence.

We move now to $1 \mathrm{D}$ structures. For $1 \mathrm{D}$ ribbons we are interested in particular in the time evolution of zero-energy edge states. As shown in Figures 2 and 3 Floquet edge states exist only in a narrow portion of the $1 \mathrm{D} \mathrm{BZ}$ where we now plot their time evolution (Figure 5). By looking at the site composition of the wave function we can unambiguously identify states localized either at the upper or at the lower edge and in the following we describe the time evolution of each of them.

Let us focus first on circular polarization. The high frequency regime (Figure 5, panels (a) and (b)) is particularly interesting; during the time evolution the two edge states modify their k-dispersion keeping however the same positive or negative slope: positive for states localized at the upper edge and negative for those localized at the lower one. This is remarkable since it corresponds to unidirectional edge states (right-movers on the lower edge and left-movers on the upper one for clockwise circular polarization) that would carry, if occupied, a constant current around the sample [33].

For a smaller frequency (Figure 5, panels (c), (d)) edge states exhibit a more complicated $\mathrm{k}$-dispersion due to overlapping Floquet bands. In this frequency regime edge states exhibit a less pronounced localization extending down into the ribbon. As it can be noticed, in this regime some additional states appear as satellites in addition to the other bands which were present at higher frequencies, too. Attention must be paid in this circumstance: these additional states, indeed, which appear at k-points far from the center of the BZ, are not proper edge states. In fact, analyzing their density distribution over time it can be seen that these are simply different itinerant states across the ribbon which at different times localize close to (but not exactly onto) the edges of the ribbon. This is also the reason why sometimes (Figure 5 panel (c)) their velocity is opposite to the one of the bona-fide edge states.

A more detailed analysis can be performed calculating the time-dependent expectation value of the velocity operator [34]

$$
\mathbf{v}_{\mathbf{k}}(t)=-i\left\langle\psi_{\mathbf{k}}(\mathbf{r}, t)|[\widehat{\mathbf{r}}, \widehat{H}(t)]| \psi_{\mathbf{k}}(\mathbf{r}, t)\right\rangle,
$$

in this way obtaining a velocity vector field in real space whose time average turns out to be

$$
\begin{aligned}
\mathbf{v}_{i}= & \sum_{i^{\prime} \alpha} \sum_{n m} \sum_{\mathbf{k}} C_{i^{\prime} n}^{\alpha *}(\mathbf{k}) C_{i m}^{\alpha}(\mathbf{k}) e^{-i \mathbf{k} \cdot\left(\mathbf{r}_{i}-\mathbf{r}_{i^{\prime}}\right)}\left(\mathbf{r}_{i}-\mathbf{r}_{i^{\prime}}\right) \\
& \cdot J_{i i^{\prime}} \frac{1}{T} \int_{-T / 2}^{T / 2} e^{-i \mathbf{A}(t) \cdot\left(\mathbf{r}_{i}-\mathbf{r}_{i^{\prime}}\right)} e^{-i(n-m) \Omega t} d t .
\end{aligned}
$$

The results for a ribbon under circularly polarized fields of different strengths and frequencies are shown in Figure 6. Edge electrons are characterized by a negligible average velocity across the ribbon (y-component) and by finite $\mathrm{x}$-components of opposite sign at the two edges, confirming their right-mover/left-mover character.

The effect of linearly polarized fields is significantly different: edge states are confined in a reduced $k$-space region and their overall dispersion is drastically reduced by orders of magnitude with respect to the case of circular polarization. Moreover, and even more notably, the right-mover/left-mover behaviour is lost and for any value of frequency and intensity the two edge bands have a parabolic dispersion, with both positive and negative slope.

Under the influence of the oscillating field we expect the charge to oscillate across the ribbon. The charge density at each site $i$ in the unit cell is calculated as the expectation 


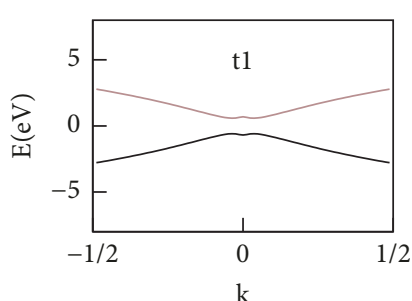

- band 1

— band 2

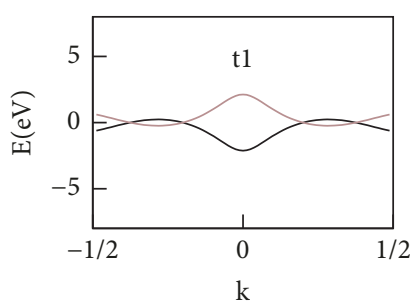

- band 1

— band 2



— band 1

— band 2

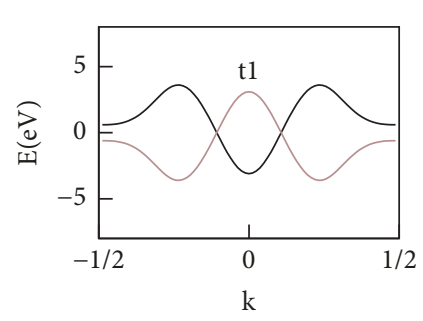

— band 1

— band 2
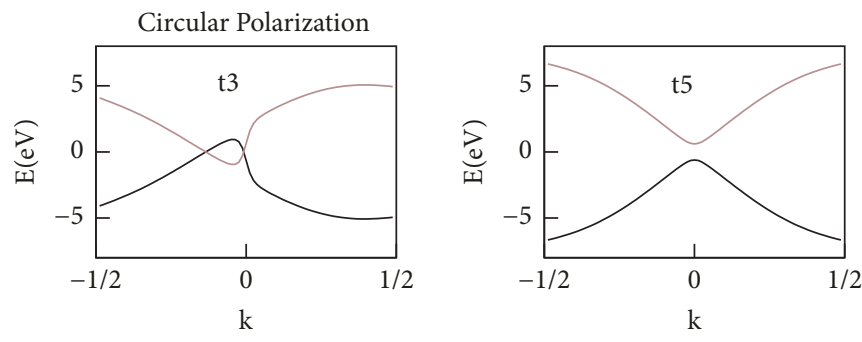

(a)
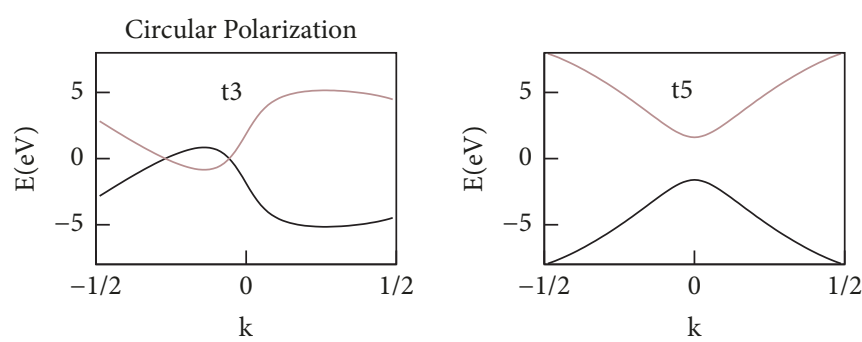

(b)
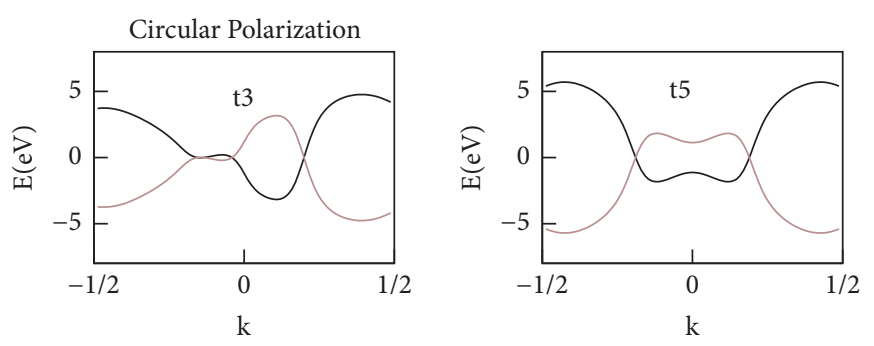

(c)
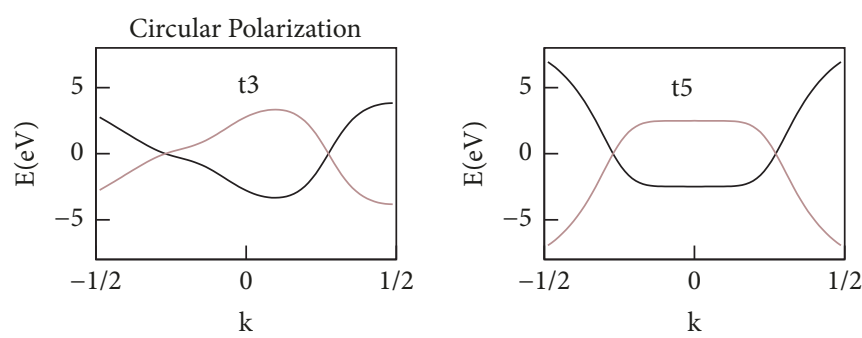

(d)

FIGURE 4: Time evolution of the band energies of 2D graphene under a circularly polarized field. Bands are plotted around the Dirac point reported as $\mathbf{k}=0$. Panels (a) and (b) refer to high frequency regime $(\Omega=12 \mathrm{eV})$ for intensities $A_{0}=0.5$ and $A_{0}=1$, respectively. Panels (c) and (d) report the results for $\Omega=5.5 \mathrm{eV}$ and $A_{0}=0.5, A_{0}=1$, respectively. Different snapshots at $t_{n}=T(n-1) / 8, T$ being the period of the external field, are shown. 

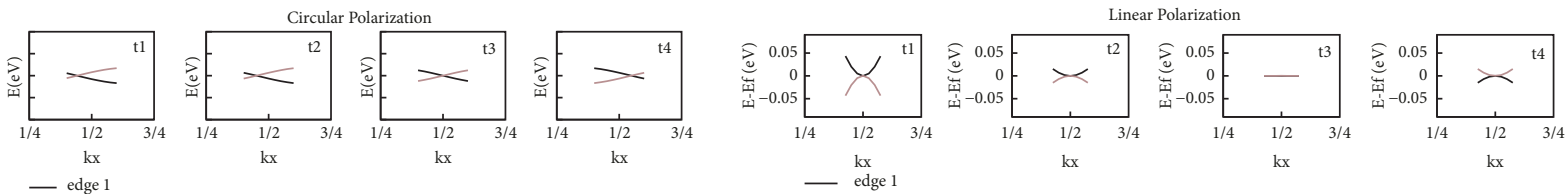

— edge 1

(a)
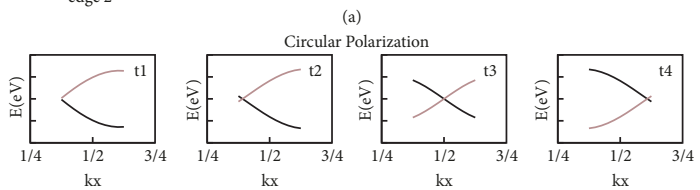

— edge 1

(b)
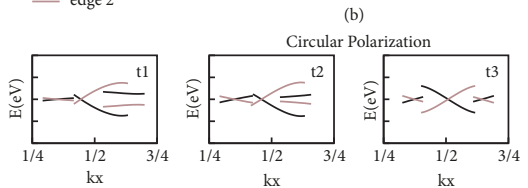

olarizatio

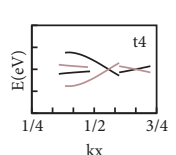

- edge 1
- edge 2
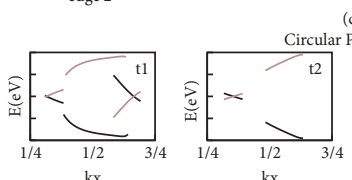

(c)

Polarization

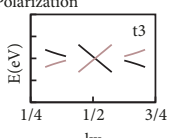

— edge

$\mathrm{kx}$

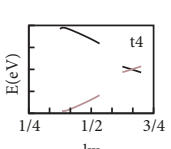

$\mathrm{kx}$

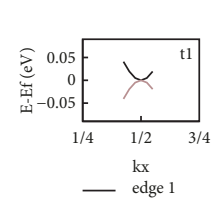

(a)

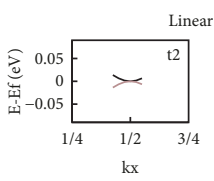

ar Polarization
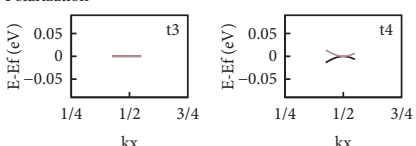

- edge 1

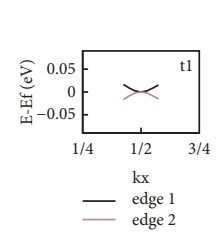

(b')

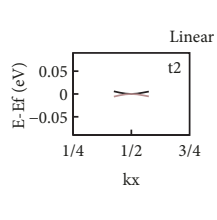

Polarization
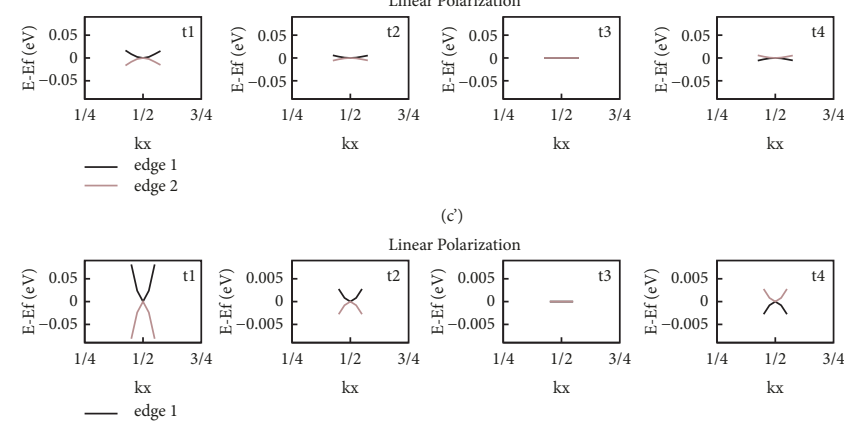

Polarization
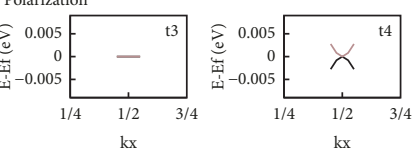

- edge 1

$\left(\mathrm{d}^{\prime}\right)$

FIGURE 5: Time evolution of the edge states of a zig-zag ribbon for circular (left panel) and linear polarization (right panel). States localized at upper and lower edge are indicated by black and brown lines, respectively. Panels (a) and (a'): $\Omega=12 \mathrm{eV}$ and $A_{0}=0.5$; panels (b) and (b'): $\Omega=12 \mathrm{eV}$ and $A_{0}=1$; panels (c) and (c'): $\Omega=5.5 \mathrm{eV}$ and $A_{0}=0.5$; panels (d) and (d'): $\Omega=5.5 \mathrm{eV}$ and $A_{0}=1$. Snapshots at times $t_{n}=T(n-1) / 8$ are reported, $T$ being the period of the external field.
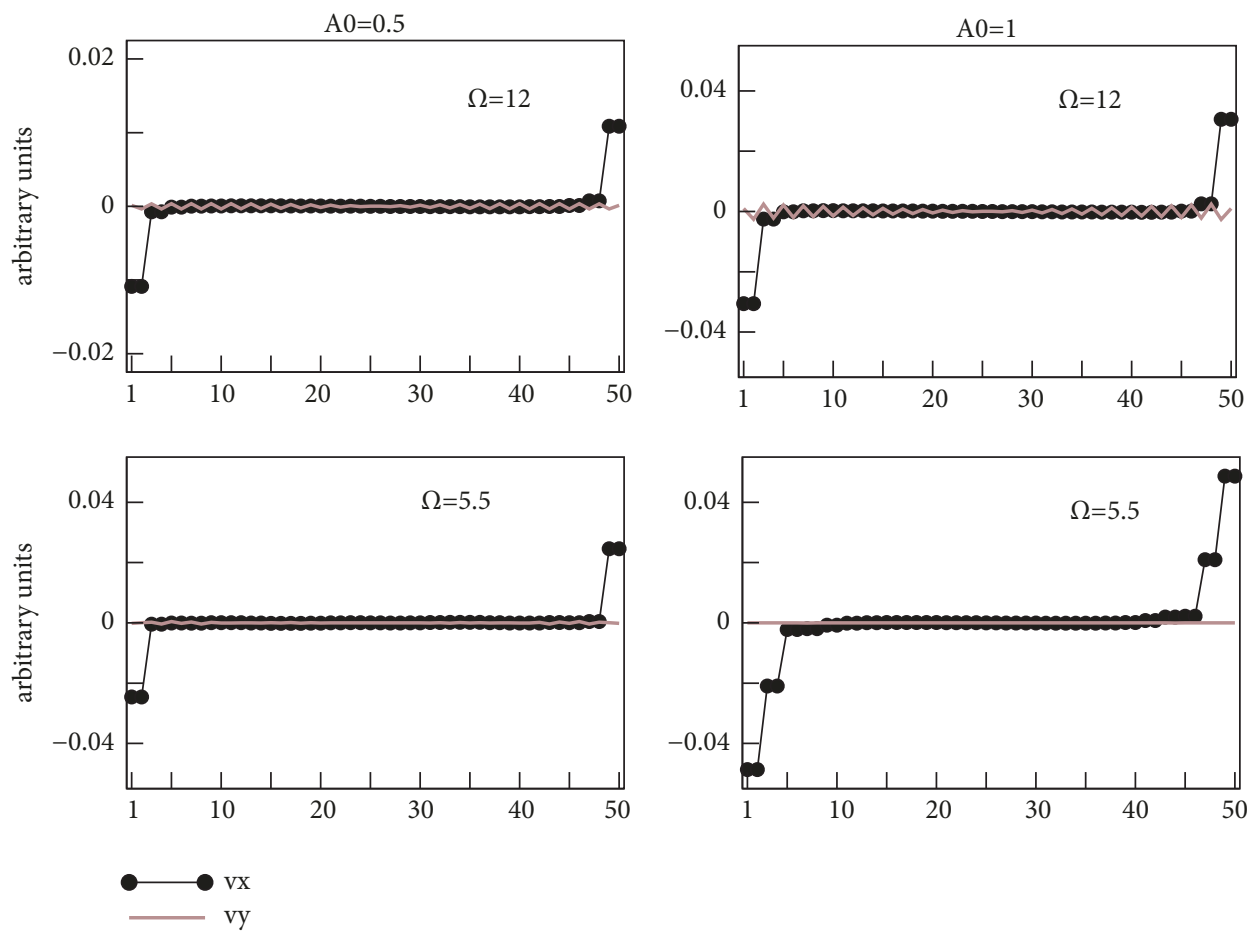

FIGURE 6: Time average of the $x$ and $y$ components of the velocity (see (10)) at ribbon sites (edges correspond to sites 1 and 50) under circularly polarized fields of different strengths and frequencies. 
value of the charge density operator $\widehat{c}_{i}^{\dagger}(t) \widehat{c}_{i}(t)$ over the manybody ground state. Since we are dealing with noninteracting electrons, the many-body states are Slater determinants which contain in the ground state single particle orbitals that correspond to the lowest energies $E_{\alpha \mathbf{k}}\left(t_{0}\right)$. It is useful to start by writing the expectation value of the charge density operator in the Heisenberg picture (from here on, we use the subscript letter $\mathrm{H}$ to indicate the Heisenberg picture, while we consider the Schroedinger picture otherwise)

$$
\rho_{i}\left(t, t_{0}\right)={ }_{H}\left\langle\Psi_{0}^{N}\left|\widehat{c}_{i}^{\dagger}(t)_{H} \widehat{c}_{i}(t)_{H}\right| \Psi_{0}^{N}\right\rangle_{H},
$$

which can be rewritten in the Schrödinger picture as follows (the details of the calculations are given in the Appendix):

$$
\begin{aligned}
& \rho_{i}\left(t, t_{0}\right) \\
& \quad=\left\langle\Psi_{0}^{N}\left(t_{0}\right)\left|\widehat{U}^{\dagger}\left(t, t_{0}\right) \widehat{c}_{i}^{\dagger}(t) \widehat{c}_{i}(t) \widehat{U}\left(t, t_{0}\right)\right| \Psi_{0}^{N}\left(t_{0}\right)\right\rangle .
\end{aligned}
$$

In this picture, the creation and annihilation operators $\widehat{c}_{i}^{\dagger}(t)$ and $\widehat{c}_{i}(t)$ contain only the intrinsic time-periodic dependence. Moreover, we observe that the time evolution of the initial $N$-particles ground state, $\widehat{U}\left(t, t_{0}\right)\left|\Psi_{0}^{N}\left(t_{0}\right)\right\rangle$, in general is not formed by the sum of the $N$ lowest energy states at time $t$ and does depend upon $t_{0}$. We thus obtain (see the Appendix)

$$
\begin{aligned}
\rho_{i} & \left(t, t_{0}\right) \\
& =\sum_{\alpha, \mathbf{k}, n, m} e^{i(n-m) \Omega t} C_{i n}^{\alpha *}(\mathbf{k}) C_{i m}^{\alpha}(\mathbf{k}) \theta\left(E_{F}-E_{\alpha \mathbf{k}}\left(t_{0}\right)\right),
\end{aligned}
$$

where the step function involves the energy $E_{\alpha, \mathbf{k}}\left(t_{0}\right)$ and guarantees that only the single particle orbitals that at time $t_{0}$ give rise to the lowest total energy are involved. This corresponds to a "quasi-equilibrium" occupation of Floquet bands at $t=t_{0}=0$ : this is physically motivated by the fact that when the Floquet quasi-steady states are formed, their occupation is stationary in time, which does not mean that the time-dependent energies are statically occupied as in equilibrium, due to the intrinsic time-periodic dependence. $E_{F}$ is the highest occupied level that satisfies the correct electron counting.

Since the initial time $t_{0}$ is arbitrary, an average over $t_{0}$ is required and we get

$$
\bar{\rho}_{i}(t)=\frac{1}{T} \int_{0}^{T} \rho_{i}\left(t, t_{0}\right) d t_{0} .
$$

The above definition starts from a strong assumption concerning the initial state condition: the electrons are assumed to be from the beginning in a quasi-equilibrium steady state associated with the presence of a pulse of "infinite" duration or rather of duration much larger than the oscillation period. In order to check the validity of this assumption we have tested a different initial condition corresponding to a very rapid switching on of the field. According to this "sudden approximation" the initial state is the unperturbed ground state $\left|\Phi_{0}^{(0)}\right\rangle$ (i.e., the solution of the undriven static Hamiltonian $\widehat{H}_{0}$, namely, $\left.\widehat{H}_{0}\left|\Phi_{0}^{(0)}\right\rangle=E_{0}\left|\Phi_{0}^{(0)}\right\rangle\right)$, and the time evolution is described as projecting $\left|\Phi_{0}^{(0)}\right\rangle$ onto the Floquet quasi-steady states and evolving time-periodically from then on. Within this sudden approximation the charge density is given by (see the Appendix)

$$
\begin{aligned}
\rho_{i}^{\prime}(t)= & \sum_{\alpha, \mathbf{k}, n, m} C_{i, n}^{\alpha}(\mathbf{k})^{*} C_{i, m}^{\alpha}(\mathbf{k}) \\
& \cdot e^{i(n-m) \Omega t}\left\|\left\langle\Phi_{0}^{(0)}(t) \mid \Psi_{\alpha, \mathbf{k}}(t)\right\rangle\right\| .
\end{aligned}
$$

The results of these two approaches are shown in Figure 7 for circular and linear polarization. We see that, in the large frequency regime and for the relatively small field intensity considered here, the two results are substantially coincident; i.e., the quasi-equilibrium and the sudden approximation are consistent with each other. This is explained by the intrinsically ultrafast dynamics induced by the laser: the charge oscillations and energy dynamics are fast enough (this is guaranteed in the driving frequency regime that is considered) to prevent states to relax and occupy lower energy levels when they are above the corresponding equilibrium Fermi level. In all cases we find that excess charge accumulates at the ribbon edges and moves in time from one edge to the other.

The width of charge oscillations at the edges is more pronounced for linear polarization and grows with field intensity, in agreement with physical intuition. The situation is more complicated for circular polarization where the strongest oscillations occur for $\Omega=5.5 \mathrm{eV}$ and $A_{0}=0.5$ (panel (c) of Figure 7). For the same frequency but for higher intensity ((panel (d) of Figure 7) we notice less charge accumulating at the edges. This is a consequence of the higher occupation of bands corresponding to itinerant states which are less or not localized, resulting into an overall less localization of the noninteracting ground state.

This analysis allows us to conclude that fields of appropriate frequency and intensity of both linear and circular polarization induce an oscillating dipole across the ribbon. This approach can be extended to the expectation values of the current density [1] and then to the calculation within the Floquet scheme of nonlinear optical properties under intense laser fields (high harmonic generation [35] and saturable absorption $[36,37]$ ) of graphene. This will be the subject of further study.

In summary, we have shown how the time evolution of physical observables in systems driven out of equilibrium by a time-periodic electromagnetic field can be obtained from Floquet eigenstates and eigenvalues. In graphene ribbons the effects depend strongly on the polarization of the applied field and in the case of circularly polarized light in a given regime of frequency and intensity unidirectional edge states are identified that describe electrons moving in opposite directions along the edges. The expectation values of the time-dependent Hamiltonian over the time-dependent single particle wave functions represent an extension of the band structure to the time domain giving information on the time evolution of single particle energies and on their population and to physical quantities that require a summation over occupied states such as charge density. These time-dependent single particle energies are essential in order to study the time evolution of the ground state energy of a collection of independent 

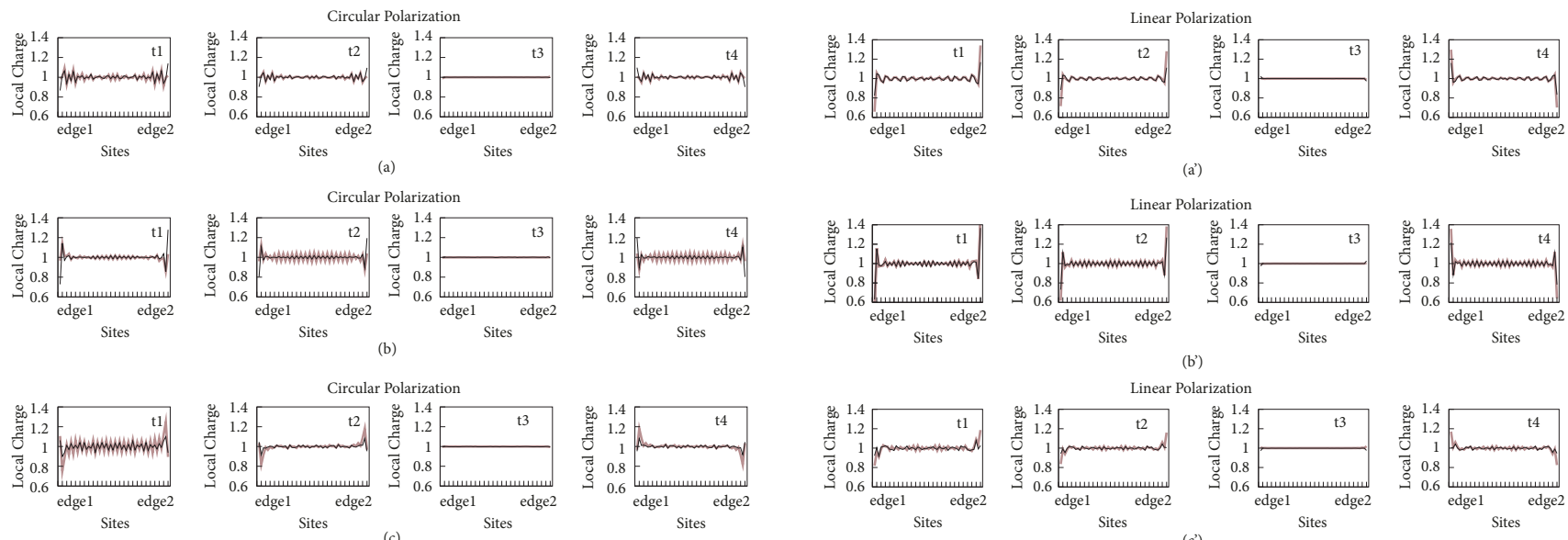

Polarization
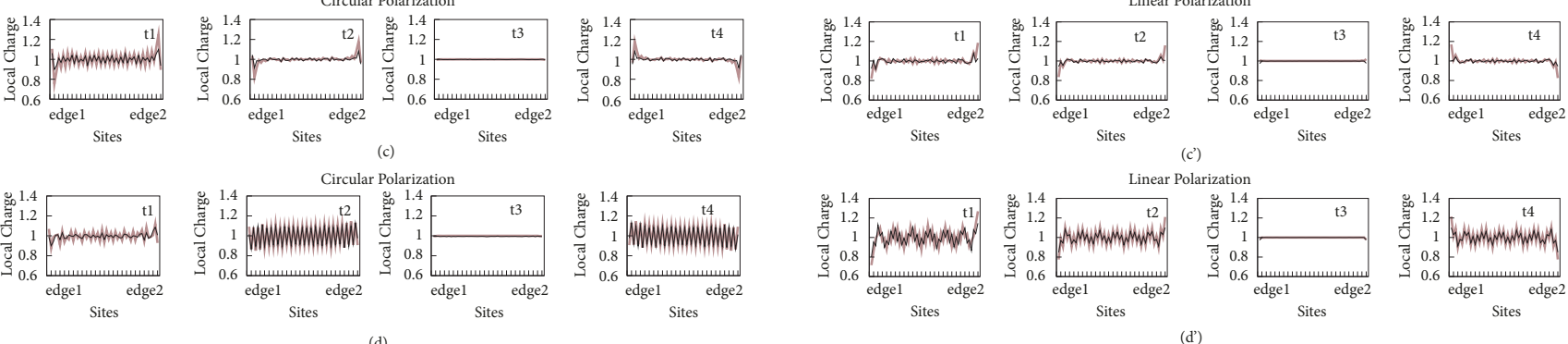

$\left(c^{\prime}\right)$
Polarization
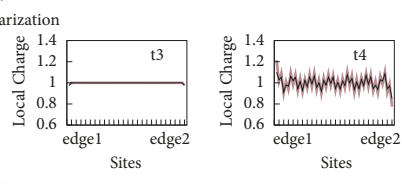

Figure 7: Time evolution of the local charge calculated for circularly (left panels) and linearly (right panels) polarized fields of different strengths and frequencies. In black are the results for $\bar{\rho}_{i}(t)$ of (14) and in brown are the results for $\rho_{i}^{\prime}(t)$ of (15) (see text). Panels (a) and (a'): $\Omega=12 \mathrm{eV}$ and $A_{0}=0.5$. Panels (b) and (b'): $\Omega=12 \mathrm{eV}$ and $A_{0}=1$. Panels (c) and (c'): $\Omega=5.5 \mathrm{eV}$ and $A_{0}=0.5$. Panels (d) and (d'): $\Omega=5.5$ $\mathrm{eV}$ and $A_{0}=1$. Snapshots are given at times $t_{n}=T(n-1) / 8$.

particles and consequently of the ground state expectation values of physical observables. A part from the results that we have obtained for a specific system, graphene ribbon in the high frequency off-resonant regime, this analysis has fundamental implications related to state population and ground state definition that are ineluctable for the correct evaluation of physical quantities in driven quantum systems.

\section{Appendix}

In order to describe our time-periodic Hamiltonian in second quantized form we consider the Hilbert space which consists of site-localized time-periodic functions $\phi_{i, n}(r, t)=$ $\phi_{i}(r) e^{-i n \Omega t}$, the so-called Sambe space [38]. The second quantized form of the Hamiltonian reads

$$
\begin{aligned}
\widehat{H}= & \sum_{i, j} \sum_{n=-\infty}^{+\infty} \sum_{m=-\infty}^{+\infty} \\
& \cdot\left\langle\phi_{i}(r)|H(r, t)| \phi_{j}(r)\right\rangle e^{-i(n-m) \Omega t} \widehat{c}_{i, n}^{\dagger} \widehat{c}_{j, m}
\end{aligned}
$$

where the matrix elements of the Hamiltonian are given in (7) of the main text. According to [39] we define

$$
\begin{aligned}
\widehat{c}_{i}(t) & =\sum_{n=-\infty}^{+\infty} e^{i n \Omega t} \widehat{c}_{i, n}, \\
\widehat{c}_{i}^{\dagger}(t) & =\sum_{n=-\infty}^{+\infty} e^{-i n \Omega t} \widehat{c}_{i, n}^{\dagger} .
\end{aligned}
$$

Finally the following is obtained [40]:

$$
\begin{aligned}
\widehat{H}(t) & =\sum_{i, j} J_{i, j} e^{-i \vec{A}(t) \cdot\left(\vec{r}_{i}-\vec{r}_{j}\right)} \widehat{c}_{i}^{\dagger}(t) \widehat{c}_{j}(t) \\
& =\sum_{i, j} J_{i, j} e^{-\overrightarrow{i A}(t) \cdot\left(\vec{r}_{i}-\vec{r}_{j}\right)} e^{-i(n-m) \Omega t} \widehat{c}_{i, n}^{\dagger} \widehat{c}_{j, m} .
\end{aligned}
$$

The charge density at time $t$ has been defined in (11) as

$$
\rho_{i}\left(t, t_{0}\right)={ }_{H}\left\langle\Psi_{0}^{N}\left|\widehat{c}_{i}^{\dagger}(t)_{H} \widehat{c}_{i}(t)_{H}\right| \Psi_{0}^{N}\right\rangle_{H} .
$$

Here states and the operators are written in the Heisenberg picture. The connection with the Schrödinger picture is the standard one:

$$
\begin{aligned}
\left|\Psi_{0}^{N}\right\rangle_{H} & =\widehat{U}^{\dagger}\left(t, t_{0}\right)\left|\Psi_{0}^{N}(t)\right\rangle, \\
\widehat{O}_{H}(t) & =\widehat{U}^{\dagger}\left(t, t_{0}\right) \widehat{O}(t) \widehat{U}\left(t, t_{0}\right),
\end{aligned}
$$

where $\widehat{O}(t)$ is a generic operator in the Schrödinger picture.

Substituting the previous expressions into (A.5), we get

$$
\begin{aligned}
\rho_{i}\left(t, t_{0}\right) & \\
= & \left(\left\langle\Psi_{0}^{N}(t)\right| \widehat{U}\left(t, t_{0}\right)\right)\left(\widehat{U}^{\dagger}\left(t, t_{0}\right) \widehat{c}_{i}^{\dagger}(t) \widehat{U}\left(t, t_{0}\right)\right) \\
& \cdot\left(\widehat{U}^{\dagger}\left(t, t_{0}\right) \widehat{c}_{i}(t) \widehat{U}\left(t, t_{0}\right)\right)\left(\widehat{U}^{\dagger}\left(t, t_{0}\right)\left|\Psi_{0}^{N}(t)\right\rangle\right), \\
= & \left\langle\Psi_{0}^{N}(t)\left|\widehat{c}_{i}^{\dagger}(t) \widehat{c}_{i}(t)\right| \Psi_{0}^{N}(t)\right\rangle .
\end{aligned}
$$

Furtherly, making use of the evolution of the Schrödinger states

$$
\left|\Psi_{0}^{N}(t)\right\rangle=\widehat{U}\left(t, t_{0}\right)\left|\Psi_{0}^{N}\left(t_{0}\right)\right\rangle
$$


we can write

$$
\begin{aligned}
\rho_{i}\left(t, t_{0}\right)= & \left\langle\Psi_{0}^{N}\left(t_{0}\right)\right| \widehat{U}^{\dagger}\left(t, t_{0}\right) \widehat{c}_{i}^{\dagger}(t) \widehat{c}_{i}(t) \widehat{U}\left(t, t_{0}\right) \\
& \cdot\left|\Psi_{0}^{N}\left(t_{0}\right)\right\rangle,
\end{aligned}
$$

which coincides with (12).

Let us consider this expression with $N=1$ in order to drop the index $N$ for simplicity of notation (the same reasoning can be applied for any $N$ ):

$$
\begin{aligned}
\rho_{i}\left(t, t_{0}\right)= & \left(\left\langle\Psi_{0}\left(t_{0}\right)\right| \widehat{U}^{\dagger}\left(t, t_{0}\right) \widehat{c}_{i}^{\dagger}(t)\right) \\
& \cdot\left(\widehat{c}_{i}(t) \widehat{U}\left(t, t_{0}\right)\left|\Psi_{0}\left(t_{0}\right)\right\rangle\right) .
\end{aligned}
$$

The ket can be rewritten as follows:

$$
\begin{aligned}
& \widehat{c}_{i}(t) \widehat{U}\left(t, t_{0}\right)\left|\Psi_{0}\left(t_{0}\right)\right\rangle=\sum_{n, \alpha, \mathbf{k}} e^{i n \Omega t} C_{i, n}^{\alpha}(\mathbf{k}) \\
& \cdot \widehat{c}_{\alpha, \mathbf{k}} \widehat{U}\left(t, t_{0}\right)\left|\Psi_{0}\left(t_{0}\right)\right\rangle=\sum_{n, \alpha, \mathbf{k} \alpha^{\prime}, \mathbf{k}^{\prime}} e^{i n \Omega t} C_{i, n}^{\alpha}(\mathbf{k}) \\
& \cdot \widehat{c}_{\alpha, \mathbf{k}}\left|\psi_{\alpha^{\prime}, \mathbf{k}^{\prime}}(t)\right\rangle\left\langle\psi_{\alpha^{\prime}, \mathbf{k}^{\prime}}(t)\left|\widehat{U}\left(t, t_{0}\right)\right| \Psi_{0}\left(t_{0}\right)\right\rangle \\
& =\sum_{n, \alpha, \mathbf{k}} e^{i n \Omega t} C_{i, n}^{\alpha}(\mathbf{k}) \widehat{c}_{\alpha, \mathbf{k}}\left|\psi_{\alpha, \mathbf{k}}(t)\right\rangle\left\langle\psi_{\alpha, \mathbf{k}}(t)\right| \widehat{U}\left(t, t_{0}\right) \\
& \cdot\left|\Psi_{0}\left(t_{0}\right)\right\rangle=\sum_{n, \alpha, \mathbf{k}} e^{i n \Omega t} C_{i, n}^{\alpha}(\mathbf{k}) \widehat{c}_{\alpha, \mathbf{k}}\left|\psi_{\alpha, \mathbf{k}}(t)\right\rangle \\
& \cdot\left\langle\psi_{\alpha, \mathbf{k}}\left(t_{0}\right) \mid \Psi_{0}\left(t_{0}\right)\right\rangle .
\end{aligned}
$$

Here we have used the identity decomposition 》 = $\sum_{\alpha^{\prime}, \mathbf{k}^{\prime}}\left|\psi_{\alpha^{\prime}, \mathbf{k}^{\prime}}(t)\right\rangle\left\langle\psi_{\alpha^{\prime}, \mathbf{k}^{\prime}}(t)\right|$ and the expansion of the annihilation operator in the k-dependent Floquet-Bloch eigenstates $\widehat{c}_{i}(t)=\sum_{n, \alpha, \mathbf{k}} e^{i n \Omega t} C_{i, n}^{\alpha}(\mathbf{k}) \widehat{c}_{\alpha, \mathbf{k}}$.

Repeating the same procedure for the term $\left\langle\Psi_{0}\left(t_{0}\right)\right| \widehat{U}^{\dagger}\left(t, t_{0}\right) \widehat{c}_{i}^{\dagger}(t)$, we get

$$
\begin{gathered}
\left\langle\Psi_{0}\left(t_{0}\right)\right| \widehat{U}^{\dagger}\left(t, t_{0}\right) \widehat{c}_{i}^{\dagger}(t)=\sum_{m, \beta, \mathbf{K}} e^{-i m \Omega t} C_{i, m}^{\beta *}(\mathbf{K}) \\
\cdot\left\langle\Psi_{0}\left(t_{0}\right) \mid \psi_{\beta, \mathbf{K}}\left(t_{0}\right)\right\rangle\left\langle\psi_{\beta, \mathbf{K}}(t)\right| \widehat{c}_{\beta, \mathbf{K}}^{\dagger} .
\end{gathered}
$$

Substituting expressions (A.12) and (A.11) into (A.10), we get

$$
\begin{aligned}
\rho_{i}( & \left(t, t_{0}\right)=\sum_{n, \alpha, \mathbf{k}} \sum_{m, \beta, \mathbf{K}} e^{i(n-m) \Omega t} C_{i, m}^{\beta *}(\mathbf{K}) C_{i, n}^{\alpha}(\mathbf{k}) \\
\cdot & \left\langle\Psi_{0}\left(t_{0}\right) \mid \psi_{\beta, \mathbf{K}}(t)\right\rangle\left\langle\psi_{\beta, \mathbf{K}}(t)\left|\widehat{c}_{\beta, \mathbf{K}}^{\dagger} \widehat{c}_{\alpha, \mathbf{k}}\right| \psi_{\alpha, \mathbf{k}}(t)\right\rangle \\
\cdot & \left\langle\psi_{\alpha, \mathbf{k}}\left(t_{0}\right) \mid \Psi_{0}\left(t_{0}\right)\right\rangle=\sum_{n, m, \alpha, \mathbf{k}} e^{i(n-m) \Omega t} C_{i, m}^{\alpha *}(\mathbf{k}) \\
\cdot & C_{i, n}^{\alpha}(\mathbf{k})\left\langle\Psi_{0}\left(t_{0}\right) \mid \psi_{\alpha, \mathbf{k}}\left(t_{0}\right)\right\rangle\left\langle\psi_{\alpha, \mathbf{k}}\left(t_{0}\right) \mid \Psi_{0}\left(t_{0}\right)\right\rangle \\
= & \sum_{n, m, \alpha, \mathbf{k}} e^{i(n-m) \Omega t} C_{i, m}^{\alpha *}(\mathbf{k}) C_{i, n}^{\alpha}(\mathbf{k}) \\
\cdot & \left|\left\langle\Psi_{0}\left(t_{0}\right) \mid \psi_{\alpha, \mathbf{k}}\left(t_{0}\right)\right\rangle\right|^{2},
\end{aligned}
$$

where we used the orthogonality of the Bloch waves to obtain $\delta_{\mathbf{k}, \mathbf{K}} \delta_{\alpha, \beta}$ and get rid of the indices $\beta, \mathbf{K}$. In addition, since it is $\left|\left\langle\Psi_{0}\left(t_{0}\right) \mid \psi_{\alpha, \mathbf{k}}\left(t_{0}\right)\right\rangle\right|^{2}=\theta\left(E_{F}-E_{\alpha, \mathbf{k}}\left(t_{0}\right)\right)$, we end up with

$$
\begin{aligned}
& \rho_{i}\left(t, t_{0}\right) \\
& =\sum_{n, m, \alpha, \mathbf{k}} e^{i(n-m) \Omega t} C_{i, m}^{\alpha *}(\mathbf{k}) C_{i, n}^{\alpha}(\mathbf{k}) \theta\left(E_{F}-E_{\alpha, \mathbf{k}}\left(t_{0}\right)\right),
\end{aligned}
$$

as in (13).

Analogously, we can perform a similar procedure using the unperturbed ground state $\left|\Phi_{0}^{(0)}\right\rangle$ (solution of the Schrödinger equation with the undriven Hamiltonian $\widehat{H}_{0}$, i.e., $\left.\widehat{H}_{0}\left|\Phi_{0}^{(0)}\right\rangle=E_{0}\left|\Phi_{0}^{(0)}\right\rangle\right)$, in order to obtain the bracket expression $\left\langle\Phi_{0}^{(0)}\left(t_{0}\right)\left|\widehat{U}^{\dagger}\left(t, t_{0}\right)\right| \Psi_{\alpha, \mathbf{k}}(t)\right\rangle$ where $\widehat{U}^{\dagger}\left(t, t_{0}\right)$ contains the rapid switch-on of the driving (in the sudden approximation) and the rest of the time evolution, ending up with

$$
\begin{aligned}
\rho_{i}^{\prime}(t)= & \sum_{\alpha, \mathbf{k}, n, m} C_{i, n}^{\alpha}(\mathbf{k})^{*} C_{i, m}^{\alpha}(\mathbf{k}) \\
& \cdot e^{i(n-m) \Omega t}\left\|\left\langle\Phi_{0}^{(0)}(t) \mid \Psi_{\alpha, \mathbf{k}}(t)\right\rangle\right\| .
\end{aligned}
$$

\section{Data Availability}

The data used to support the findings of this study are available from the corresponding author upon request.

\section{Disclosure}

Part of the results of the present study have been presented at the 2017 Spring Meeting of the Deutsche Physikalische Gesellschaft in Dresden.

\section{Conflicts of Interest}

The authors declare that they have no conflicts of interest.

\section{References}

[1] F. H. Faisal and J. Z. Kamiński, "Floquet-Bloch theory of highharmonic generation in periodic structures," Physical Review A: Atomic, Molecular and Optical Physics, vol. 56, no. 1, pp. 748$762,1997$.

[2] M. Grifoni and P. Hanngi, "Driven quantum tunneling," Physics Reports, vol. 304, no. 5-6, pp. 229-354, 1998.

[3] J. H. Shirley, "Solution of the Schrödinger Equation with a Hamiltonian Periodic in Time," Physical Review A: Atomic, Molecular and Optical Physics, vol. 138, no. 4B, pp. B979-B987, 1965.

[4] V. Dal Lago, M. Atala, and L. E. Foa Torres, "Floquet topological transitions in a driven one-dimensional topological insulator," Physical Review A: Atomic, Molecular and Optical Physics, vol. 92, no. 2, 2015.

[5] J. Cayssol, B. Dóra, F. Simon, and R. Moessner, "Floquet topological insulators," Physica Status Solidi - Rapid Research Letters, vol. 7, no. 1-2, pp. 101-108, 2013.

[6] N. Goldman, J. C. Budich, and P. Zoller, "Topological quantum matter with ultracold gases in optical lattices," Nature Physics, vol. 12, no. 7, pp. 639-645, 2016. 
[7] M. A. Sentef, M. Claassen, A. F. Kemper et al., "Theory of Floquet band formation and local pseudospin textures in pumpprobe photoemission of graphene," Nature Communications, vol. 6, 2015.

[8] N. H. Lindner, G. Refael, and V. Galitski, "Floquet topological insulator in semiconductor quantum wells," Nature Physics, vol. 7, no. 6, pp. 490-495, 2011.

[9] T. Oka and H. Aoki, "Erratum: Photovoltaic Hall effect in graphene (Physical Review B - Condensed Matter and Materials Physics (2009) 79 (081406))," Physical Review B: Condensed Matter and Materials Physics, vol. 79, no. 16, 2009.

[10] M. S. Rudner, N. H. Lindner, E. Berg, and M. Levin, "Anomalous Edge States and the Bulk-Edge Correspondence for Periodically Driven Two-Dimensional Systems," Physical Review X, vol. 3, no. 3, 2013.

[11] G. Usaj, P. M. Perez-Piskunow, L. E. F. Foa Torres, and C. A. Balseiro, "Irradiated graphene as a tunable Floquet topological insulator," Physical Review B: Condensed Matter and Materials Physics, vol. 90, no. 11, 2014.

[12] A. Farrell and T. Pereg-Barnea, "Photon-Inhibited Topological Transport in Quantum Well Heterostructures," Physical Review Letters, vol. 115, no. 10, 2015.

[13] A. Kundu, H. A. Fertig, and B. Seradjeh, "Floquet-Engineered Valleytronics in Dirac Systems," Physical Review Letters, vol. 116, no. 1, 2016.

[14] Z. Gu, H. A. Fertig, D. P. Arovas, and A. Auerbach, "Floquet Spectrum and Transport through an Irradiated Graphene Ribbon," Physical Review Letters, vol. 107, no. 21, 2011.

[15] P. M. Perez-Piskunow, G. Usaj, C. A. Balseiro, and L. E. Torres, "Floquet chiral edge states in graphene," Physical Review B: Condensed Matter and Materials Physics, vol. 89, no. 12, 2014.

[16] T. Kitagawa, T. Oka, A. Brataas, L. Fu, and E. Demler, "Transport properties of nonequilibrium systems under the application of light: Photoinduced quantum Hall insulators without Landau levels," Physical Review B: Condensed Matter and Materials Physics, vol. 84, no. 23, 2011.

[17] M. Lababidi, I. I. Satija, and E. Zhao, "Counter-propagating Edge Modes and Topological Phases of a Kicked Quantum Hall System," Physical Review Letters, vol. 112, no. 2, 2014.

[18] Z. Zhou, I. I. Satija, and E. Zhao, "Floquet edge states in a harmonically driven integer quantum Hall system," Physical Review B: Condensed Matter and Materials Physics, vol. 90, no. 20, 2014.

[19] F. D. M. Haldane, "Model for a quantum hall effect without landau levels: Condensed-matter realization of the "parity anomaly"," Physical Review Letters, vol. 61, no. 18, pp. 2015-2018, 1988.

[20] S. Reich, J. Maultzsch, C. Thomsen, and P. Ordejón, “Tightbinding description of graphene," Physical Review B: Condensed Matter and Materials Physics, vol. 66, no. 3, 2002.

[21] D. R. Hofstadter, "Energy levels and wave functions of Bloch electrons in rational and irrational magnetic fields," Physical Review B: Condensed Matter and Materials Physics, vol. 14, no. 6, pp. 2239-2249, 1976.

[22] K. Jiménez-García, L. J. LeBlanc, R. A. Williams, M. C. Beeler, A. R. Perry, and I. B. Spielman, "Peierls Substitution in an Engineered Lattice Potential," Physical Review Letters, vol. 108, no. 22, 2012.

[23] F. Manghi, C. Calandra, and E. Molinari, "Bonding and surface electronic structure of an Sb overlayer on GaP(110)," Surface Science, vol. 184, no. 3, pp. 449-462, 1987.

[24] C. L. Kane and E. J. Mele, " $Z_{2}$ topological order and the quantum spin hall effect," Physical Review Letters, vol. 95, no. 14, Article ID 146802, 2005.
[25] F. Grandi, F. Manghi, O. Corradini, and C. M. Bertoni, "Topological properties of the bond-modulated honeycomb lattice," Physical Review B: Condensed Matter and Materials Physics, vol. 91, no. 11, 2015.

[26] F. Grandi, F. Manghi, O. Corradini, C. M. Bertoni, and A. Bonini, "Topological invariants in interacting quantum spin Hall: A cluster perturbation theory approach," New Journal of Physics, vol. 17, 2015.

[27] W. Yao, S. A. Yang, and Q. Niu, "Edge States in Graphene: From Gapped Flat-Band to Gapless Chiral Modes," Physical Review Letters, vol. 102, no. 9, 2009.

[28] U. De Giovannini, H. Hübener, and A. Rubio, "Monitoring Electron-Photon Dressing in WSe2," Nano Letters, vol. 16, no. 12, pp. 7993-7998, 2016.

[29] A. Farrell, A. Arsenault, and T. Pereg-Barnea, "Dirac cones, Floquet side bands, and theory of time-resolved angle-resolved photoemission," Physical Review B: Condensed Matter and Materials Physics, vol. 94, no. 15, 2016.

[30] F. Mahmood, C.-K. Chan, Z. Alpichshev et al., "Selective scattering between Floquet-Bloch and Volkov states in a topological insulator," Nature Physics, vol. 12, no. 4, pp. 306-310, 2016.

[31] R. Bertoncini and A. P. Jauho, "Gauge-invariant formulation of the intracollisional field effect including collisional broadening," Physical Review B: Condensed Matter and Materials Physics, vol. 44, no. 8, pp. 3655-3664, 1991.

[32] J. K. Freericks, H. R. Krishnamurthy, M. A. Sentef, and T. P. Devereaux, "Gauge invariance in the theoretical description of time-resolved angle-resolved pump/probe photoemission spectroscopy," in Proceedings of the Frontiers of Quantum and Mesoscopic Thermodynamics, FQMT 2013, Czech Republic, August 2013.

[33] J. P. Dahlhaus, B. M. Fregoso, and J. E. Moore, "Magnetization Signatures of Light-Induced Quantum Hall Edge States," Physical Review Letters, vol. 114, no. 24, 2015.

[34] M. Puviani, F. Manghi, and A. Bertoni, "Dynamics and control of edge states in laser-driven graphene nanoribbons," Physical Review B: Condensed Matter and Materials Physics, vol. 95, no. 23, 2017.

[35] J. D. Cox, A. Marini, and F. J. de Abajo, "Plasmon-assisted highharmonic generation in graphene," Nature Communications, vol. 8, p. 14380, 2017.

[36] A. Marini, J. D. Cox, and F. J. de Abajo, "Nonperturbative theory of graphene saturable absorption," in Proceedings of the 2017 Conference on Lasers and Electro-Optics Europe (CLEO/Europe) \& European Quantum Electronics Conference (EQEC), pp. 1-1, Munich, June 2017.

[37] Z. Sun, T. Hasan, F. Torrisi et al., "Graphene mode-locked ultrafast laser," ACS Nano, vol. 4, no. 2, pp. 803-810, 2010.

[38] H. Sambe, "Steady states and quasienergies of a quantum-mechanical system in an oscillating field," Physical Review A: Atomic, Molecular and Optical Physics, vol. 7, no. 6, pp. 22032213, 1973.

[39] A. Gómez-León and G. Platero, "Floquet-Bloch Theory and Topology in Periodically Driven Lattices," Physical Review Letters, vol. 110, no. 20, 2013.

[40] P. Delplace, Á. Gómez-León, and G. Platero, "Merging of Dirac points and Floquet topological transitions in ac-driven graphene," Physical Review B: Condensed Matter and Materials Physics, vol. 88, no. 24, 2013. 

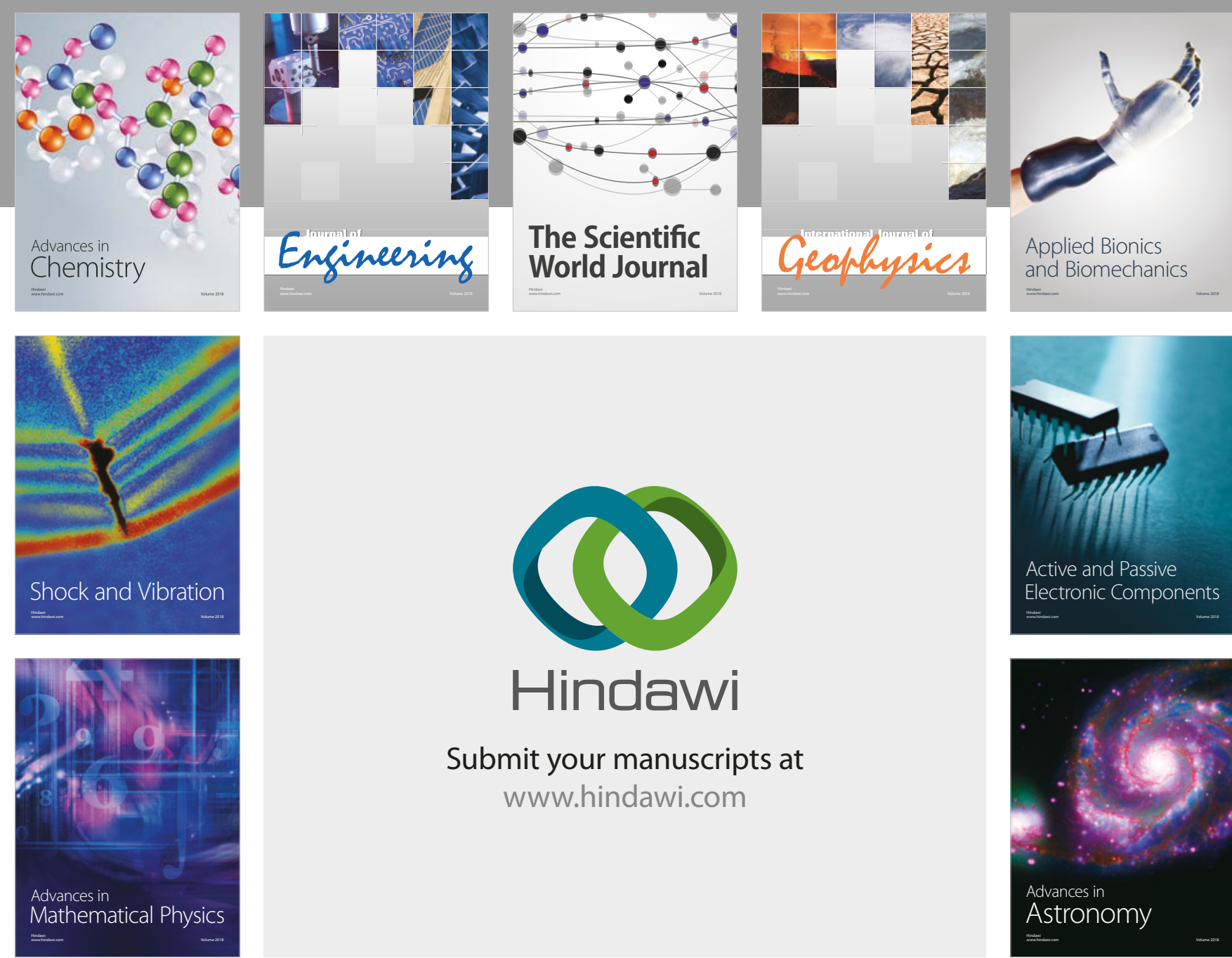

Submit your manuscripts at

www.hindawi.com

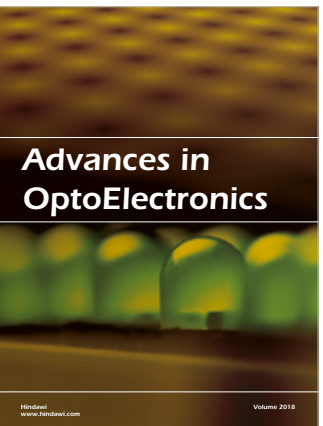

\section{Rotcting Machinery}
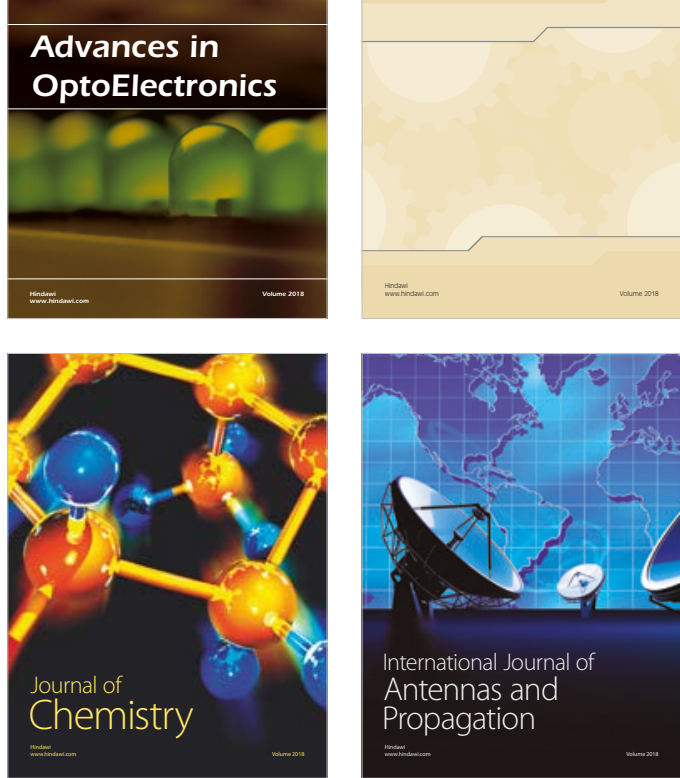

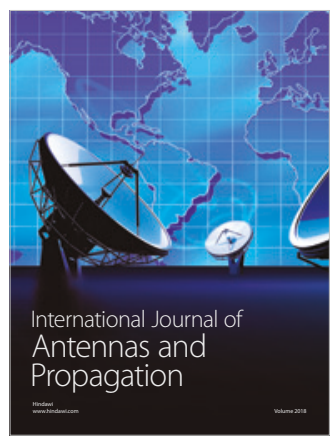

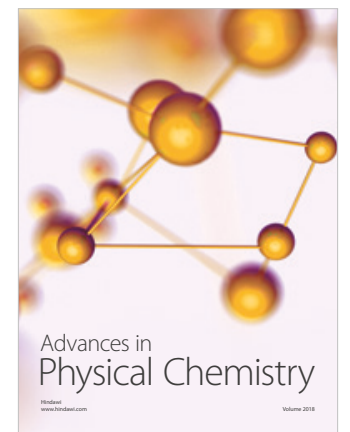

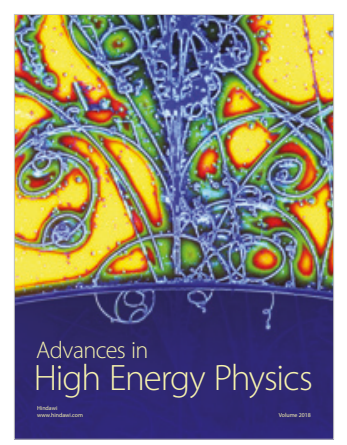

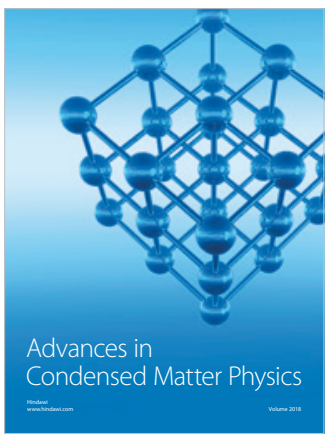

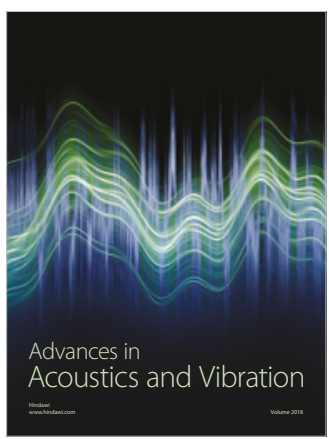

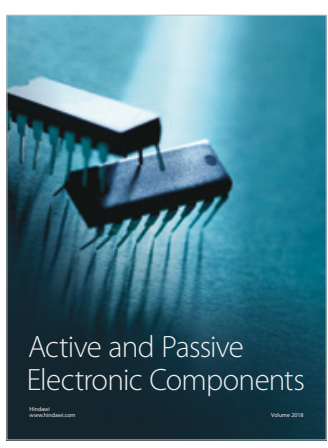
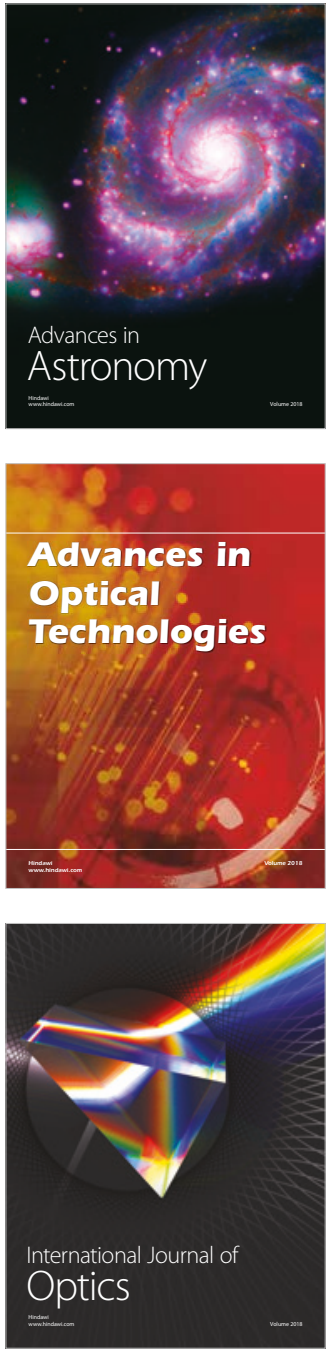